\title{
Fatores associados ao consumo de bebidas açucaradas entre pré-escolares brasileiros: inquérito nacional de 2006
}

\author{
Factors associated with the consumption of soft drinks \\ among Brazilian preschool children: national survey of 2006
}

Juliana Bergamo Vega ${ }^{1}$

Ana Paula Poblacion ${ }^{1}$

José Augusto de Aguiar Carrazedo Taddei ${ }^{1}$

${ }^{1}$ Departamento de Pediatria, Universidade Federal de São Paulo. R. Loefgren 1647, Vila Clementino. 04040-032 São Paulo SP Brasil.

jvbergamo@hotmail.com
Abstract The rising consumption of sweetened beverages such as soft drinks or artificial juices is associated with the prevalence of obesity in Brazil and around the world. This study seeks to verify the frequency of consumption of these beverages among Brazilian children aged 24-59 months and to investigate the association of soft drinks with demographic, socioeconomic and nutritional variables. Using data from the National Survey on Demography and Health of Women and Children - 2006, the eating habits were obtained using the food and drink frequency questionnaire for the seven days preceding the interview, and anthropometry recorded the weight and height of children. Among preschoolers, 37.3\% consumed soft drinks and artificial juices 4 or more days per week. The factors significantly associated with frequent consumption of soft drinks were living in regions of higher economic development of the country, in urban areas, belonging to the higher income bracket, with mothers watching TV regularly and excess weight of the child, were associated with consumption of soft drinks and artificial juices 4 or more days per week. Further studies are needed to understand the effective contribution of soft drinks on the epidemic of childhood obesity.

Key words Soft drinks, Fruit juices, Carbonated soft drinks, Socioeconomic factors, Obesity, Preschool children, Epidemiological surveys
Resumo O crescente consumo de bebidas açucaradas como os refrigerantes ou sucos artificiais está associado à prevalência de obesidade no Brasil e no mundo. Este estudo se propõe a conhecer a frequência de consumo destas bebidas entre crianças brasileiras de 24 a 59 meses e investigar associações com variáveis demográficas, socioeconômicas e nutricionais. Utilizando dados da Pesquisa Nacional de Demografia e Saúde da Criança e da Mulher-2006, o padrão alimentar foi obtido com o questionário de frequência de alimentos e bebidas nos sete dias anteriores à data da entrevista e a antropometria usou medidas de peso e de estatura das crianças. Entre os pré-escolares, 37,3\% consumiram refrigerantes e sucos artificiais 4 dias ou mais por semana. Os fatores que apresentaram associação estatisticamente significante com consumo frequente de $B A$ foram residir em regiões de maior desenvolvimento econômico do país, em áreas urbanas, pertencer ao estrato de maior poder aquisitivo, mães que assistiam TV regularmente e excesso de peso da criança, estavam associados ao consumo de refrigerantes e sucos artificiais em 4 ou mais dias por semana. Novos estudos são necessários para a compreensão da real contribuição das bebidas açucaradas na epidemia de obesidade infantil.

Palavras-chave Refrigerantes, Sucos, Bebidas Gasosas, Fatores Socioeconômicos, Obesidade, Pré -Escolar, Inquéritos Epidemiológicos. 


\section{Introdução}

Mudanças dos padrões alimentares nas últimas décadas, com aumento marcante no consumo de industrializados, mais especificamente das bebidas açucaradas (BA), como os refrigerantes e sucos artificiais, vêm sendo apontadas como o principal contribuinte para o aumento da energia total das dietas. A ingestão excessiva de calorias, observadas em idades cada vez mais precoces, influencia o ganho de peso e o aparecimento de marcadores inflamatórios, podendo ser considerado um dos fatores ambientais determinantes para as proporções epidêmicas da obesidade no mundo ${ }^{1-6}$.

A prevalência de obesidade em crianças e adolescentes mais que duplicou nos Estados Unidos nas últimas três décadas ${ }^{7}$. Entre brasileiros de 2 a 5 anos a prevalência de excesso de peso passou de $3 \%$, em 1989, para $7,7 \%$, em $2006^{8}$. Paralelamente, entre 1975 e 2003, o consumo per capita de refrigerantes no Brasil aumentou o equivalente a $490 \%$, assemelhando-se ao mercado americano que apresentou crescimento de 500\% nos últimos 50 anos $^{9,10}$. Em 2006, 22,1\% das crianças de 6 a 59 meses consumiam diariamente ${ }^{11}$, e $70 \%$, ao menos uma vez por semana refrigerantes ou sucos artificiais no país ${ }^{12}$.

Em alguns países desenvolvidos, os consumidores frequentes de BA, entre 2 e 5 anos, são predominantemente de famílias com menores rendas e escolaridade ${ }^{13-16}$. No entanto, em países em desenvolvimento como o Brasil, ainda são escassos os estudos com representatividade nacional que busquem investigar as possíveis variáveis que se associam a este padrão de consumo apenas no público pré-escolar, faixa etária que teve crescimento expressivo de excesso de peso entre os inquéritos de 1996 e $2006^{8}$.

A relevância do tema para a promoção da saúde na infância e suas consequências na idade adulta, justifica a realização deste estudo que objetiva conhecer as prevalências do consumo de BA entre pré-escolares e identificar as variáveis socioeconômicas, demográficas e nutricionais que se associam a esse consumo.

\section{Desenho do estudo}

Utilizaram-se dados secundários da Pesquisa Nacional de Demografia e Saúde da Criança e da Mulher (PNDS) realizada no ano 2006. Esta pesquisa caracteriza-se por ser um inquérito domiciliar, com amostragem probabilística comple$\mathrm{xa}$, de representatividade nacional. As unidades amostrais foram selecionadas em dois estágios sendo a primeira denominada unidades primárias, compostas por setores censitários, e a segunda as unidades secundárias formadas por domicílios randomicamente selecionados nos setores sorteados. Foram considerados para o universo do estudo, domićlios particulares, em setores comuns ou não especiais, incluindo favelas, selecionados em dez estratos amostrais, representativo das cinco macrorregiões brasileiras e dos contextos urbano e rural. Os dados completos do inquérito, bem como o detalhamento da sua metodologia estão disponíveis no site http://bvsms. saude.gov.br/bvs/pnds/index.php ${ }^{17}$.

\section{Elegibilidade e critério de seleção}

Foram coletados dados de aproximadamente 15 mil mulheres de 15 a 49 anos, e 5.000 crianças menores, de 59 meses de idade, filhos biológicos das entrevistadas e destas, 4.817 viviam com suas mães na ocasião do inquérito. Das 2.915 crianças de 24 a 59 meses de idade foram excluídas 34, devido à falta de informações no banco de dados em relação ao consumo de refrigerantes e sucos artificiais, totalizando 2.881 pré-escolares.

\section{Variável dependente}

A frequência semanal do consumo de BA foi obtida por meio do questionário da frequência de alimentos (QFA). A PNDS investigou vinte alimentos e bebidas quanto a sua frequência de consumo nos 7 dias que antecederam a data da entrevista. Foram consideradas as seguintes possibilidades de respostas: não consumiu, consumiu 1 dia por semana, de 2 a 3 dias por semana, de 4 a 6 dias por semana e todos os dias da semana. Este estudo analisou o item denominado "refrigerante e sucos artificiais". Para as análises a variável foi categorizada em três níveis de consumo: infrequente ( 1 ou menos dias por semana), regular ( 2 a 3 dias por semana) e frequente (4 dias ou mais por semana).

\section{Variáveis independentes}

Idade (em meses): categorizada em 24 a 35, 36 a 47 e de 48 a 59 meses completos para análise descritiva e em 24 a 41 meses e 42 a 59 meses para análises inferenciais.

Sexo: feminino e masculino.

Macrorregião administrativa: categorizada em Norte (N), Nordeste (Ne), Centro-Oeste (CO), Sudeste (Su) e Sul (S) para a análise des- 
critiva e regiões de maior desenvolvimento econômico $(\mathrm{CO}+\mathrm{Su}+\mathrm{S})$ e menor desenvolvimento econômico do país $(\mathrm{N}+\mathrm{Ne})$ para análises inferenciais.

Situação de domicílio: urbano ou rural.

Classificação econômica: utilizou-se o sistema de pontuação padronizado do Critério de Classificação Econômica Brasil (CCEB), versão 2008, que quantifica oito bens duráveis do domicílio e o número de empregados mensalistas, além do grau de instrução do chefe da família, gerando pontos de corte para a determinação do poder aquisitivo da pessoa ou da família ${ }^{18}$. A partir disso, estima-se a condição econômica para o domicílio. Para a análise descritiva, agregou-se em cinco (A, B, C, D e E) dos oito estratos sugeridos na proposta original dos pontos de corte da Associação Brasileira de Empresas de Pesquisa (2007/2008). Para as análises inferenciais esta variável foi dicotomizada em classes econômicas de maior poder aquisitivo $(\mathrm{A}+\mathrm{B}+\mathrm{C})$ e menor poder aquisitivo (classe $\mathrm{D}+\mathrm{E})$.

Exposição da mãe à TV: A PNDS de 2006 questionou a mãe quanto ao "costume de assistir televisão" com as seguintes possibilidades de resposta: "todos os dias", "quase todos os dias", "pelo menos 1 x por semana", "menos de $1 \mathrm{x}$ por mês" e "não vê". A fim de investigar se a exposição frequente à televisão se associa ao consumo de BA, optou-se por categorizar em maior frequência (mães que responderam assistir todos os dias e quase todos os dias) e menor frequência (mães que responderam assistir pelo menos $1 \mathrm{x}$ por semana, menos de 1 x por mês ou não assistiam).

Escolaridade Materna: categorizada em 0 a 4, 5 a 8 e 9 ou mais anos de estudo para a análise descritiva, e de 0 a 8 anos de estudo ( $\leq 8$ anos) e mais de 8 anos de estudo ( $>8$ anos) para as análises inferenciais.

\section{Estado Nutricional}

Os equipamentos antropométricos, treinamento e padronização dos entrevistadores e a supervisão e controle de qualidade das medidas aferidas foram de responsabilidade do Laboratório de Avaliação Nutricional de Populações do Departamento de Nutrição da Faculdade de Saúde Pública da Universidade de São Paulo. As técnicas utilizadas na obtenção de todas as medidas seguiram procedimentos padronizados segundo Lohman et al. ${ }^{19}$ e foram tomadas em duplicata, adotando-se como resultado final a média das duas medidas.

Utilizou-se o indicador de peso para estatura $(\mathrm{P} / \mathrm{E})$, expresso em escore $\mathrm{Z}$, de acordo com os critérios estabelecidos pela Organização Mundial da Saúde (OMS) ${ }^{20}$. Para caracterização da amostra as crianças foram categorizadas de acordo com as classificações: magreza e magreza acentuada, valores de $\mathrm{P} / \mathrm{E}$ menores de -2 escore $Z$; eutrofia, valores entre -2 e +2 escore Z; sobrepeso, valores entre +2 escore $Z ; \leq 3$ escore $Z$; e obesidade, valores maiores de +3 escore Z. Para os modelos de regressão, optou-se por comparar eutróficos com sobrepeso e obesos, este último de forma agrupada. Para esta análise, não foram consideradas as crianças que apresentaram magreza e magreza extrema $(n=16)$.

\section{Análise dos dados}

As análises foram feitas com o pacote estatístico Stata / IC 12 (StataCorp LP, College Station, TX, USA). A fim de considerar os procedimentos amostrais, utilizou-se o efeito do plano amostral nas análises. Os pesos da amostra foram aplicados apenas nas estatísticas descritivas ${ }^{17}$.

Nas análises descritivas utilizou-se o teste qui-quadrado, considerando significantes valores de $p<0,05$. Nas análises inferenciais optou-se por utilizar o modelo de regressão logística multinomial, que permite avaliar desfechos politômicos (três ou mais categorias). Este tipo de modelo possibilitou a comparação das categorias "consumo regular" ( 2 - 3 dias por semana) e "consumo frequente" (4 dias ou mais por semana) de maneira independente com a categoria de referência "consumo infrequente" (1 dia ou menos por semana). Nas regressões simples foram consideradas as variáveis com $\mathrm{p}<0,20 \mathrm{da}$ categoria de interesse "consumo frequente" (4 dias ou mais por semana), elegíveis para inclusão no modelo de regressão múltipla. A estratégia adotada para ordem de inclusão das variáveis no modelo foi o forward selection. Permaneceram no modelo final as variáveis com $\mathrm{p}<0,05$ da categoria de interesse "consumo frequente" (4 dias ou mais por semana) $)^{21}$.

\section{Aspectos Éticos}

Os dados da PNDS foram coletados segundo os critérios estabelecidos pela Declaração de Helsinki, tendo todos os procedimentos envolvendo seres humanos aprovados pelo Comitê de Ética do Centro de Referência e Treinamento em DST / AIDS, do Departamento de Saúde do Estado de São Paulo. Em adição, este trabalho foi aprovado pelo Comitê de Ética em Pesquisa da Universidade Federal de São Paulo / Hospital São Paulo. 


\section{Resultados}

Em 2006, mais que um terço dos pré-escolares brasileiros, representando quase três milhões de crianças, consumiram BA 4 dias ou mais dos 7 dias que antecederam a realização da pesquisa. As maiores prevalências de consumo frequente de BA foram observadas na faixa etária de 36 a 47 meses $(37,2 \%)$, nas regiões Sudeste $(41,4 \%)$ e Sul $(51,0 \%)$, em regiões urbanas do país $(39,4 \%)$, nos estratos de maior poder aquisitivo C $(41,6 \%)$, B $(41,1 \%)$ e A $(39,3 \%)$, entre filhos de mães que assistiam televisão frequentemente $(36,7 \%)$, e que tinham entre 5 e 8 anos de estudo $(38,9 \%)$ e entre os desvios nutricionais magreza e magreza acentuada $(50,0 \%)$, sobrepeso $(43,0 \%)$ e obesidade (49,2\%). Em adição, 8,2\% das crianças apresentavam excesso de peso, sendo 5,9\% com sobrepeso e 2,3\% com obesidade (Tabela 1).

Na Tabela 2, observa-se que as variáveis independentes apresentaram razões de chance diferentes, de acordo com a frequência do consumo de BA. Viver em regiões urbanas (OR 1,30; IC95\% 1,02-1,64) e ser filho de mãe que assistia frequentemente televisão (OR 1,79 IC95\% 1,272,52 ) se associou de maneira independente ao consumo de BA, de 2 a 3 dias por semana. As variáveis "Macrorregiões", "Classes econômicas ABEP" e "Estado nutricional" não se associaram ao consumo regular de BA, mas se mostraram associadas à categoria "Consumo frequente" no modelo múltiplo.

As crianças que residiam nas regiões Sul, Sudeste e Centro-Oeste (OR 2,13; IC95\% 1,662,72), em regiões urbanas (OR 1,81; IC95\% 1,39$2,34)$, que pertenciam às classes $\mathrm{A}+\mathrm{B}+\mathrm{C}(\mathrm{OR}$ 1,44; IC95\% 1,15-1,81), filhos de mães que assistiam frequentemente televisão (OR 1,90; IC95\% $1,35-2,68)$ e que apresentavam excesso de peso (OR 1,41; IC95\% 1,02-1,99), tinham mais risco de serem consumidores frequentes de BA.

\section{Discussão}

Por este estudo, utilizar amostra de representatividade nacional e ter o rigor da padronização da coleta bem como a credibilidade das instituições responsáveis pela execução do projeto pode-se inferir confiabilidade dos dados.

Por outro lado, inquéritos transversais possuem limitações ao inferir causalidade ou relação temporal. Outra limitação deste estudo refere-se ao instrumento utilizado para coletar informações de consumo alimentar, o QFA, o qual coleta apenas o número de dias em que foram ingeridos os alimentos e bebidas sem quantificá-los. Em geral, instrumentos que registram o consumo de alimentos estão sujeitos a erros aleatórios devido, por exemplo, à dificuldade dos indivíduos em lembrar dos alimentos consumidos, interferindo na precisão dos dados coletados ${ }^{22}$. Vale ressaltar ainda que o item "refrigerantes e sucos artificiais" não representa a totalidade de bebidas açucaradas disponíveis no mercado, que são habitualmente consumidas pelas crianças como, por exemplo, as bebidas esportivas, aquelas à base de soja e as lácteas com adição de açúcar. Quanto aos fatores associados, foi possível analisar as variáveis disponíveis no banco de dados. Entretanto, há outros determinantes de consumo de BA referidos pela literatura que não foram investigados no inquérito que serviu de base para este estudo, como, por exemplo, acesso e disponibilidade destes produtos para esta população.

As escolhas alimentares são determinadas por variáveis biológicas, socioeconômicas, demográficas e culturais em um processo dinâmico, que varia de acordo com o contexto e o estágio da vida do indivíduo ${ }^{1,23}$. Conforme os resultados encontrados neste artigo, pré-escolares que viviam em regiões de maior desenvolvimento econômico apresentavam duas vezes mais chance de consumirem frequentemente BA. Aqueles que pertenciam a famílias de maior poder aquisitivo tinham $44 \%$ mais chance de serem consumidores frequentes de tais bebidas. Outro estudo brasileiro identificou maior participação percentual dos refrigerantes no valor energético total em domicílios da região Sul do país, com rendimento mensal acima de 30 salários mínimos, quando comparada à região Norte ${ }^{1}$. Ambos os achados indicam que a renda é um dos fatores determinantes para o consumo de BA e a sua distribuição desigual é representada também por limites geográficos desenhados pela história econômica do país.

Em alguns estudos realizados em países desenvolvidos, o consumo frequente de BA está associado a menor renda, menor educação dos pais e menor status econômico das famílias ${ }^{13-16}$. Tais diferenças sugerem que os determinantes socioeconômicos das escolhas alimentares assumem características diferentes entre países de maior ou menor desenvolvimento.

O processo de transição nutricional é consequência do desenvolvimento econômico, das mudanças tecnológicas e culturais que ocorreram nos últimos anos. As principais alterações na estrutura da dieta foram influenciadas pelo processo de urbanização e do aumento da renda. 
Tabela 1. Distribuição da frequência de consumo de bebidas açucaradas por crianças de 24 a 59 meses de idade segundo variáveis nutricionais, socioeconômicas e demográficas. Pesquisa Nacional de Demografia e Saúde da Criança e da Mulher, Brasil, $2006(\mathrm{n}=2.881)$.

\begin{tabular}{|c|c|c|c|c|c|c|}
\hline \multirow{2}{*}{ Variável } & \multicolumn{2}{|c|}{ Total da amostra } & \multirow{2}{*}{$\begin{array}{c}\text { Consumo } \\
\text { Infrequente } \\
(\leq 1 \text { dias/sem }) \\
\%(\% \mathrm{IC})\end{array}$} & \multirow{2}{*}{$\begin{array}{c}\text { Consumo } \\
\text { Intermediário } \\
(2-3 \text { dias/sem }) \\
\%(\% \mathrm{IC})\end{array}$} & \multirow{2}{*}{$\begin{array}{c}\text { Consumo } \\
\text { Frequente } \\
(\geq 4 \text { dias/sem }) \\
\%(\% \mathrm{IC})\end{array}$} & \multirow{2}{*}{$\begin{array}{l}\mathbf{p} \\
\chi^{2}\end{array}$} \\
\hline & $\mathrm{N}^{\mathrm{a}}$ & $\%(\% \mathrm{IC})^{\mathrm{b}}$ & & & & \\
\hline Total de crianças ${ }^{c}$ & 2.881 & $100(100)$ & $35,2(32,1-38,5)$ & $27,5(24,7-30,6)$ & $37,2(33,5-41,2)$ & \\
\hline Expansão amostral & 8.032 .400 & & 2.830 .900 & 2.209 .868 & 2.991 .632 & \\
\hline Idade (em meses) & & & & & & 0,0347 \\
\hline $24-35$ & 964 & $33,5(31,9-35,1)$ & $42,0(38,8-45,3)$ & $26,8(24,1-29,6)$ & $31,2(28,3-34,3)$ & \\
\hline $36-47$ & 975 & $33,8(32,2-35,5)$ & $38,3(35,0-41,6)$ & $24,5(21,8-27,4)$ & $37,2(34,4-58,0)$ & \\
\hline $48-59$ & 942 & $32,7(31,1-34,3)$ & $40,9(37,5-44,3)$ & $23,2(20,6-26,2)$ & $35,9(32,6-39,3)$ & \\
\hline Sexo & & & & & & 0,4072 \\
\hline Feminino & 1.483 & $48,5(46,7-50,3)$ & $41,3(38,5-44,3)$ & $25,0(22,8-27,4)$ & $33,6(31,0-36,4)$ & \\
\hline Masculino & 1.398 & $51,5(49,7-53,3)$ & $39,5(36,6-42,3)$ & $24,7(22,5-27,1)$ & $35,9(33,1-38,7)$ & \\
\hline Macrorregiões & & & & & & $<0,0001$ \\
\hline Norte & 592 & $20,6(19,1-22,1)$ & $49,3(43,6-55,0)$ & $24,7(20,9-28,9)$ & $26,0(21,5-31,1)$ & \\
\hline Nordeste & 548 & $19,0(17,7-20,4)$ & $44,3(39,5-49,3)$ & $34,3(30,2-38,7)$ & $21,4(17,6-25,7)$ & \\
\hline Sudeste & 596 & $20,7(19,4-22,0)$ & $33,6(28,9-38.6)$ & $25,0(21,1-29,3)$ & $41,4(36,5-46,5)$ & \\
\hline Sul & 563 & $19,5(18,3-20,9)$ & $32,5(28,3-37,0)$ & $16,5(13,7-19,81)$ & $51,0(46,2-55,7)$ & \\
\hline Centro-Oeste & 582 & $20,2(18,9-21,6)$ & $42,1(37,3-47,1)$ & $24,0(20,6-27,9)$ & $33,9(29,4-38,6)$ & \\
\hline Situação de domicílio & & & & & & $<0,0001$ \\
\hline Urbano & 1.845 & $64,0(60,4-67,5)$ & $35,2(32,7-37,9)$ & $25,4(23,3-27,6)$ & $39,4(36,8-42,1)$ & \\
\hline Rural & 1.036 & $36,0(32,5-39,6)$ & $49,5(45,4-53,6)$ & $23,9(21,1-27,0)$ & $26,6(23,1-30,3)$ & \\
\hline $\mathrm{ABEP}^{\mathrm{d}}$ & & & & & & $<0,0001$ \\
\hline A & 107 & $3,7(3,0-4,6)$ & $42,1(32,4-52,3)$ & $18,7(12,4-27,2)$ & $39,2(29,8-49,6)$ & \\
\hline $\mathrm{B}$ & 521 & $18,1(16,5-19,8)$ & $35,5(31,2-40,1)$ & $23,4(19,9-27,4)$ & $41,1(36,7-45,6)$ & \\
\hline $\mathrm{C}$ & 1.104 & $38,3(36,1-40,6)$ & $34,5(31,5-37,7)$ & $23,9(21,4-26,6)$ & $41,6(38,4-44,8)$ & \\
\hline $\mathrm{D}$ & 759 & $26,4(24,5-28,3)$ & $41,1(37,1-45,3)$ & $28,1(24,6-31,8)$ & $30,8(27,2-34.8)$ & \\
\hline $\mathrm{E}$ & 390 & $13,5(11,8-15,5)$ & $61,5(55,0-67,7)$ & $24,9(19,9-30,6)$ & $13,6(9,7-18,8)$ & \\
\hline Exposição à televisão & & & & & & $<0,0001$ \\
\hline Frequente $^{\mathrm{e}}$ & 2.528 & $87,8(85,8-89,4)$ & $37,9(35,7-40,1)$ & $25,4(23,6-27,3)$ & $36,7(34,5-38,9)$ & \\
\hline Infrequente $^{\mathrm{f}}$ & 353 & $12,2(10,6-14,2)$ & $58,3(51,5-64,9)$ & $20,7(16,4-25,8)$ & $21,0(16,2-26,7)$ & \\
\hline Escolaridade Materna (anos) & & & & & & $<0,0001$ \\
\hline $0-4$ & 832 & $29,1(26,8-31,5)$ & $50,7(46,4-55,0)$ & $22,7(19,6-26,2)$ & $26,6(22,9-30,6)$ & \\
\hline $5-8$ & 1.012 & $35,4(33,4-37,4)$ & $36,9(33,5-40,3)$ & $24,2(21,5-27,1)$ & $38,9(35,6-42,4)$ & \\
\hline$\geq 9$ & 1.016 & $35,5(33,3-37,8)$ & $35,4(32,3-38,7)$ & $27,3(24,53-30,2)$ & $37,3(34,2-40,6)$ & \\
\hline Estado Nutricional (P/E) & & & & & & 0,0363 \\
\hline Magreza e Magreza acentuada & 16 & $0,6(0,4-1,0)$ & $37,5(17,9-62,3)$ & $12,5(3,1-38,6)$ & $50,0(27,2-72,8)$ & \\
\hline Eutrofia & 2.393 & $91,2(90,0-92,2)$ & $40,8(38,3-43,3)$ & $25,3(23,4-27,2)$ & $33,9(31,7-36,3)$ & \\
\hline Sobrepeso & 156 & $5,9(5,1-6,9)$ & $33,3(26,2-41,3)$ & $23,7(17,9-30,7)$ & $43,0(35,1-51,1)$ & \\
\hline Obesidade & 61 & $2,3(1,8-3,0)$ & $29,5(19,5-42,0)$ & $21,3(12,9-33,2)$ & $49,2(37,3-61,2)$ & \\
\hline
\end{tabular}

a Se inferior a 2.881, a diferença deve-se à inexistência das informações no banco de dados; ${ }^{\mathrm{b}} \mathrm{IC}$ : Intervalo de confiança; ${ }^{\mathrm{c}}$ Valores utilizando os pesos amostrais; ${ }^{\mathrm{A} A s s o c i a c ̧ a ̃ o ~ B r a s i l e i r a ~ d a s ~ E m p r e s a s ~ d e ~ P e s q u i s a ; ~}{ }^{\mathrm{e}}$ Frequente: Todos os dias ou quase todos os dia; ${ }^{\mathrm{f}}$ Infrequente: pelo menos $1 \mathrm{x}$ por semana, menos de $1 \mathrm{x}$ por mês ou não vê.

Em países em desenvolvimento, os padrões alimentares das regiões urbana e rural são expressivamente diferentes, principalmente devido à maior disponibilidade de alimentos processados em regiões urbanas ${ }^{24}$. Isto pôde ser observado nesta pesquisa, já que residir em regiões urbanas aumentou em $81 \%$ o risco de os consumidores infantis ingerirem frequentemente BA.
Outro fator relevante neste processo é a expansão da mídia, especialmente no meio de comunicação televisiva, que se tornou veículo promotor de hábitos alimentares pouco saudáveis e do sedentarismo ${ }^{24}$. Este estudo evidencia que, em 2006, no Brasil, as mães que referiram assistir frequentemente televisão tinham $90 \%$ mais chance de terem filhos consumidores frequentes de BA e 
Tabela 2. Associação entre categorias de consumo de bebidas açucaradas e variáveis nutricionais, socioeconômicas e demográficas. Crianças brasileiras de 24 a 59 meses de idade. Pesquisa Nacional de Demografia e Saúde da Criança e da Mulher, Brasil, 2006 ( $\mathrm{n}=2.610)$.

\begin{tabular}{|c|c|c|c|c|}
\hline \multirow{3}{*}{$\begin{array}{c}\text { Referência: } \text { Consumo infrequente } \leq 1 \mathrm{dias} / \mathrm{sem}^{*} \\
\text { Variável }\end{array}$} & \multicolumn{4}{|c|}{ Consumo regular (2-3 dias por semana) } \\
\hline & \multicolumn{2}{|c|}{ Análise bruta } & \multicolumn{2}{|c|}{ Análise ajustada ${ }^{a}$} \\
\hline & $\begin{array}{c}\mathrm{OR}^{\mathrm{b}} \\
(\mathrm{IC}-95 \%)^{\mathrm{c}}\end{array}$ & $\mathbf{p}$ & $\begin{array}{c}\mathrm{OR}^{\mathrm{b}} \\
(\mathrm{IC}-95 \%)^{\mathrm{c}}\end{array}$ & $\mathbf{p}$ \\
\hline \multicolumn{5}{|l|}{ Idade da criança (meses) } \\
\hline 24-41 meses & 1 & & & \\
\hline 42-59 meses & $1,00(0,83-1,18)$ & 0,909 & - & \\
\hline \multicolumn{5}{|l|}{ Sexo } \\
\hline Feminino & 1 & & & \\
\hline Masculino & $1,03(0,86-1,24)$ & 0,724 & - & \\
\hline \multicolumn{5}{|l|}{ Macrorregiões } \\
\hline$<$ Desenvolvimento Econômico $^{\mathrm{d}}$ & 1 & & 1 & \\
\hline$>$ Desenvolvimento Econômico ${ }^{\mathrm{e}}$ & $0,97(0,78-1,21)$ & 0,814 & $0,96(0,76-1,22)$ & 0,768 \\
\hline \multicolumn{5}{|l|}{ Situação de domicílio } \\
\hline Rural & 1 & & 1 & \\
\hline Urbano & $1,49(1,19-1,86)$ & 0,001 & $1,30(1,02-1,64)$ & 0,032 \\
\hline \multicolumn{5}{|l|}{$\mathrm{ABEP}^{\mathrm{f}}$} \\
\hline Classe $\mathrm{D}+\mathrm{E}$ & 1 & & 1 & \\
\hline Classe $\mathrm{A}+\mathrm{B}+\mathrm{C}$ & $1,18(0,95-1,47)$ & 0,125 & $1,01(0,79-1,29)$ & 0,911 \\
\hline \multicolumn{5}{|l|}{ Exposição à televisão } \\
\hline Menor frequência & 1 & & 1 & \\
\hline Todos/quase todos os dias & $1,90(1,37-2,63)$ & $<0,001$ & $1,79(1,27-2,52)$ & 0,001 \\
\hline \multicolumn{5}{|l|}{ Escolaridade materna (anos) } \\
\hline$\leq 8$ anos & 1 & & & \\
\hline$>$ 8anos & $1,41(1,14-1,74)$ & 0,002 & - & \\
\hline \multicolumn{5}{|l|}{ Estado Nutricional (escore z P/E) } \\
\hline Eutrofia & 1 & & 1 & \\
\hline Sobrepeso e Obesidade & $1,15(0,79-1,69)$ & 0,466 & $1,10(0,75-1,61)$ & 0,617 \\
\hline
\end{tabular}

79\% mais chance de ter filhos consumidores regulares de BA.

Um amplo estudo realizado pela Organização Pan-Americana de Saúde (OPAS) mostrou que a maioria das propagandas de alimentos divulga aqueles ricos em gordura, açúcar e sal e muitas são direcionadas às crianças, público especialmente vulnerável à influência da publicidade ${ }^{25}$. O marketing utilizado visa formar vínculos emocionais oferecendo, por exemplo, diversão por meio do uso de desenhos animados ou sites na internet e brindes. Sendo assim, a falta de uma regulamentação mais efetiva expõe as crianças à publicidade invasiva, que enfraquece a eficácia de qualquer aconselhamento sobre bons hábitos alimentares ${ }^{25}$. No Reino Unido, por exemplo, existem regulamentações mais rigorosas da publicidade direcionada às crianças. No Brasil, esta é uma discussão que vem ganhando força nos últimos anos e faz parte de um conjunto de medidas que visa controlar a obesidade infantil. Infelizmente, muitos projetos de lei desta natureza, ainda estão em tramitação nos órgãos competentes $^{26}$.

As bebidas açucaradas são consideradas as maiores contribuintes para o aumento de açúcar de adição e seu consumo regular é apontado com um dos fatores determinantes para o aumento de energia na dieta e ganho de peso $^{4,6}$. No Brasil de 2006, 6,6\% das crianças de 0 a 5 anos encontravam-se com excesso de peso ${ }^{27}$. Analisando apenas os pré-escolares, dos aproximadamente 8 milhões, 668 mil crianças $(8,2 \%)$ estavam com excesso de peso e estes consumiam BA com maior frequência $(1,41$; IC95\% 1,02-1,97), quando comparados aos eutróficos. Não houve associação estatisticamente significante entre estado nutricional e consumo regular destas bebidas. 
Tabela 2. continuação

\begin{tabular}{|c|c|c|c|c|}
\hline \multirow[t]{3}{*}{ Referência: Consumo infrequente $\leq 1 \mathrm{dias} / \mathrm{sem}^{*}$} & \multicolumn{4}{|c|}{ Consumo frequente ( $\geq 4$ dias por semana) } \\
\hline & \multicolumn{2}{|c|}{ Análise bruta } & \multicolumn{2}{|c|}{ Análise ajustada ${ }^{a}$} \\
\hline & $\begin{array}{c}\mathrm{OR}^{\mathrm{b}} \\
(\mathrm{IC}-95 \%)^{\mathrm{c}}\end{array}$ & $\mathbf{p}$ & $\begin{array}{c}\mathrm{OR}^{\mathrm{b}} \\
(\mathrm{IC}-95 \%)^{\mathrm{c}}\end{array}$ & $\mathbf{p}$ \\
\hline \multicolumn{5}{|l|}{ Idade da criança (meses) } \\
\hline 24-41 meses & 1 & & & \\
\hline $42-59$ meses & $1,12(0,95-1,32)$ & 0,172 & - & \\
\hline \multicolumn{5}{|l|}{ Sexo } \\
\hline Feminino & 1 & & & \\
\hline Masculino & $1,12(0,94-1,32)$ & 0,193 & - & \\
\hline \multicolumn{5}{|l|}{ Macrorregiões } \\
\hline$<$ Desenvolvimento Econômico $^{\mathrm{d}}$ & 1 & & 1 & \\
\hline$>$ Desenvolvimento Econômico $^{e}$ & $2,30(1,81-2,91)$ & $<0,001$ & $2,13(1,66-2,72)$ & $<0,001$ \\
\hline \multicolumn{5}{|l|}{ Situação de domicílio } \\
\hline Rural & 1 & & 1 & \\
\hline Urbano & $2,09(1,63-2,66)$ & $<0,001$ & $1,81(1,39-2,34)$ & $<0,001$ \\
\hline \multicolumn{5}{|l|}{$\mathrm{ABEP}^{\mathrm{f}}$} \\
\hline Classe D+E & 1 & & 1 & \\
\hline Classe $\mathrm{A}+\mathrm{B}+\mathrm{C}$ & $2,25(1,82-2,78)$ & $<0,001$ & $1,44(1,15-1,81)$ & 0,002 \\
\hline \multicolumn{5}{|l|}{ Exposição à televisão } \\
\hline Menor frequência & 1 & & 1 & \\
\hline Todos/quase todos os dias & $2,70(1,91-3,82)$ & $<0,001$ & $1,90(1,35-2,68)$ & $<0,001$ \\
\hline \multicolumn{5}{|l|}{ Escolaridade materna (anos) } \\
\hline$\leq 8$ anos & 1 & & & \\
\hline$>8$ anos & $1,36(1,11-1,67)$ & 0,003 & - & \\
\hline \multicolumn{5}{|l|}{ Estado Nutricional (escore z P/E) } \\
\hline Eutrofia & 1 & & 1 & \\
\hline Sobrepeso e Obesidade & $1,66(1,19-2,32)$ & 0,003 & $1,41(1,02-1,99)$ & 0,048 \\
\hline
\end{tabular}

"Grupo cujo consumo infrequente ( $\leq 1$ dia por semana) foi considerado como referência (categoria de comparação). ${ }^{a}$ Controlado por idade e sexo; ${ }^{\mathrm{b}} \mathrm{OR}=$ Razão de chance (odds ratio); ${ }^{\mathrm{C}} \mathrm{IC}-95 \%$ : intervalo de confiança de $95 \%$; ${ }^{\mathrm{d}}$ Norte e Nordeste; ${ }^{\mathrm{e}}$ Sul, Sudeste e Centro-oeste; ${ }^{\mathrm{f}} \mathrm{ABEP}$ : Associação Brasileira das Empresas de Pesquisa.

Embora estes resultados não permitam inferir causalidade devido às limitações do desenho do estudo e da natureza dos dados, é plausível sugerir que crianças com excesso de peso apresentavam um padrão de consumo diferente em relação a estas bebidas, quando comparadas às crianças com peso adequado, representando um possível erro alimentar desta faixa etária de maior risco nutricional.

A determinação causal entre o consumo frequente de BA e o excesso de peso em crianças e adolescentes é evidenciada em diferentes estudos nos últimos anos. Um estudo transversal, que avaliou 4.283 australianos de 2 a 16 anos, encontrou $26 \%$ mais chances de serem sobrepeso/ obesos (IC 95\% 1,03-1,53), entre aqueles que relataram consumo diário $\geq 250 \mathrm{~g}$ de $\mathrm{BA}^{15}$. Um estudo de coorte, que avaliou 9.600 americanos, encontrou $43 \%$ mais chances de serem obesos em relação aos consumidores infrequentes ou não consumidores (OR 1,43 IC 95\% 1,10-1,85) entre consumidores frequentes aos 5 anos $^{13}$. Um estudo longitudinal australiano, que avaliou 1.499 pré-escolares, encontrou crianças consumidoras regulares de BA entre as refeições aos 30 meses, tinham duas vezes mais chances de estarem com excesso de peso aos 54 meses (OR 2,4 IC 95\% 1,03-5,39), quando comparadas às não consumidoras $^{14}$. Um ensaio clínico randomizado duplo-cego acompanhou durante 18 meses crianças holandesas de 4 a 11 anos, divididas em dois grupos de observação, as que consumiam bebidas sem adição de açúcar e as que consumiam BA (104 kcal em $250 \mathrm{ml} /$ dia). Os achados mostraram evolução ponderal (z escore de IMC e gordura corporal) menor no grupo que consumiu bebidas sem açúcar ${ }^{28}$. Soma-se a essas evidências, uma revisão sistemática que identificou 
associação do consumo de BA com excesso de peso em crianças em 6 estudos transversais e 4 estudos prospectivos ${ }^{3}$. Apesar da relevância dos achados destas pesquisas, muitos não podem ser diretamente comparados a este estudo devido a diferenças no delineamento e no tratamento dos dados. No entanto, todos apontam para a associação estatisticamente significante entre frequência de consumo de BA e excesso de peso.

Não é apenas a relação de BA com excesso de peso que preocupa as organizações de saúde. Outras pesquisas apontam evidências significativas da associação com o desenvolvimento de diabetes mellitus tipo 2 e doenças cardiovasculares. Entre crianças com consumo frequente de SSB foram identificados aparecimento precoce de hiperinsulinemia, dislipidemias, hipertensão, alteração dos marcadores inflamatórios e aumento da circunferência de cintura ${ }^{4-6,29}$. Tais evidências reforçam os achados de pesquisas conduzidas no Brasil que têm ressaltado a origem fetal ou mesmo na infância de muitas doenças e agravos não transmissíveis do adulto ${ }^{30}$.

O conjunto de evidências existentes somado à plausibilidade biológica reforça as hipóteses explicativas para a relação entre consumo frequente de SSB, excesso de peso e alterações metabólicas. A energia proveniente de líquidos está associada à menor saciedade, pois os líquidos não ativam os mecanismos regulatórios de ingestão nas refeições subsequentes ${ }^{4}$. Além disso, o aumento do tamanho das porções oferecidas ${ }^{4}$ somado à presença de altas concentrações de carboidratos de rápida absorção, como a sacarose ou o xarope de milho rico em frutose $e^{5,31,32}$, podem justificar consequências tão deletérias à saúde.

Fundamental para o estabelecimento das condições de saúde do indivíduo, o hábito alimentar é estabelecido na infância ${ }^{11}$ e os alimentos industrializados como o BA vêm fazendo parte da dieta habitual infantil ${ }^{33}$. Este novo padrão de consumo pode contribuir para o aumento do risco de uma população, que está formando hábitos determinantes para sua saúde ao longo da vida.

\section{Conclusão}

A elevada prevalência do consumo frequente de BA entre os pré-escolares está fortemente associada a questões socioeconômicas. Diferentemente de países de maior desenvolvimento, aqueles residentes em zonas urbanas, nas regiões de maior desenvolvimento econômico, de estratos de maior poder aquisitivo e filhos de mães que assistiam frequentemente televisão estavam expostos a condições ambientais que favorecem o consumo frequente destas bebidas açucaradas. Finalmente, crianças com excesso de peso consomem com maior frequência BA, indicando um alerta sobre um padrão alimentar seguido por esta população de risco. Novos estudos com delineamentos que possam contribuir para o conhecimento sobre a força e a direção dessa associação são necessários para compreensão da real contribuição das bebidas açucaradas na epidemia de obesidade infantil. 


\section{Colaboradores}

JB Vega participou da concepção, delineamento, análise e interpretação dos dados, redação e revisão crítica do artigo, e aprovação da versão a ser publicada. AP Poblacion participou da concepção, delineamento, redação e revisão crítica do artigo, e aprovação da versão a ser publicada. JAAC Taddei participou da concepção, delineamento, análise e interpretação dos dados, redação e revisão crítica do artigo, e aprovação da versão a ser publicada.

\section{Referências}

1. Enes CC, Silva MV. Energy and nutrients disposal in residences: the contrast between north and south regions of Brazil. Cien Saude Colet 2009; 14(4):1267-1276.

2. Monteiro CA, Levy RB, Claro RM, Castro IR, Cannon G. Increasing consumption of ultra-processed foods and likely impact on human health: evidence from Brazil. Public Health Nutr 2011; 14(1):5-13.

3. Malik VS, Schulze MB, Hu FB. Intake of sugar-sweetened beverages and weight gain: a systematic review. Am J Clin Nutr 2006; 84(2):274-288.

4. Hu FB. Resolved: there is sufficient scientific evidence that decreasing sugar-sweetened beverage consumption will reduce the prevalence of obesity and obesity-related diseases. Obes Rev 2013; 14(8):606-619.

5. Wang JW, Mark S, Henderson M, O'Loughlin J, Tremblay A, Wortman J, Paradis G, Gray-Donald K. Adiposity and glucose intolerance exacerbate components of metabolic syndrome in children consuming sugar-sweetened beverages: QUALITY cohort study. Pediatr Obes 2013; 8(4):284-293.

6. Hu FB, Malik VS. Sugar-sweetened beverages and risk of obesity and type 2 diabetes: epidemiologic evidence. Physiol Behav 2010; 100(1):47-54.

7. Ogden CL, Carroll MD, Curtin LR, McDowell MA, Tabak CJ, Flegal KM. Prevalence of overweight and obesity in the United States, 1999-2004. JAMA 2006; 295(13):1549-1555.

8. Cocetti M, Taddei JA, Konstantyner T, Konstantyner TC, Barros Filho AA. Prevalence and factors associated with overweight among Brazilian children younger than 2 years. J Pediatr 2012; 88(6):503-508.

9. Instituto Brasileiro de Geografia e Estatística (IBGE). Pesquisa de Orçamentos Familiares (POF 2002-2003). Rio de Janeiro: IBGE; 2006.

10. Ludwig DS, Peterson KE, Gortmaker SL. Relation between consumption of sugar-sweetened drinks and childhood obesity: a prospective, observational analysis. Lancet 2001; 357(9255):505-508.

11. Bortolini GA, Gubert MB, Santos LM. Food consumption Brazilian children by 6 to 59 months of age. Cad Saude Publica 2012; 28(9):1759-1771.

12. Silva NVP, Muniz LC, Vieira MFA. Consumo de refrigerantes e sucos artificiais por crianças menores de cinco anos: uma análise da Pesquisa Nacional de Demografia e Saúde da Criança e da Mulher, 2006. Nutrire 2012; 37(2):163-173.

13. DeBoer MD, Scharf RJ, Demmer RT. Sugar-sweetened beverages and weight gain in 2- to 5-year-old children. Pediatrics 2013; 132(3):413-420.

14. Dubois L, Farmer A, Girard M, Peterson K. Regular sugar-sweetened beverage consumption between meals increases risk of overweight among preschool-aged children. J Am Diet Assoc 2007; 107(6):924-934; discussion 34-35.

15. Grimes CA, Riddell LJ, Campbell KJ, Nowson CA. Dietary salt intake, sugar-sweetened beverage consumption, and obesity risk. Pediatrics 2013; 131(1):14-21.

16. Hafekost K, Mitrou F, Lawrence D, Zubrick SR. Sugar sweetened beverage consumption by Australian children: implications for public health strategy. $B M C$ Public Health 2011; 11:950. 
17. Brasil. Ministério da Saúde (MS). Pesquisa Nacional de Demografia e Saúde da Criança e da Mulher (PNDS2006). Brasília: MS; 2006.

18. Associação Brasileira de Empresas de Pesquisa (ABEP). Critério de Classificação Econômica Brasil (CCEB). São Paulo: ABEP; 2008.

19. Lohman TG, Roche AF, Martorell R. Anthropometric standardization reference manual. Champaign: $\mathrm{Hu}$ man Kinetics Books; 1988.

20. World Health Organization (WHO). Child growth standards: length/height-for-age, weight-for-age, weightfor- lenght, weight-fot-height and body mass index-forage: methods and development. Geneve: WHO; 2006.

21. Bittencourt HR. Regressão logística politômica: revisão teórica e aplicações. Acta Scientiae 2003; 5(1):77-86.

22. Slater B, Philippi ST, Marchioni DM,Fisberg RM. Validação de Questionários de Freqüência Alimentar-QFA: considerações metodológicas. Rev Bras Epidemiol. 2003; 6(3):200-208.

23. European Food Information Council. The determinants of food choice. 2005. [acessado 2015 jun 7]. Disponível em: http://www.eufic.org/article/en/expid/ review-food-choice/

24. Mondini L, Gimeno SGA. Transição Nutricional:Significado, Determinantes e Prognóstico. In: Taddei JA, Lang RMF, Longo-Silva G, Toloni MHA, organizadores. Nutrição em Saúde Pública. Rio de Janeiro: Rubio; 2011. p. 561-576.

25. Organização Panamericana de Saúde (OPAS). Recomendações da Consulta de Especialistas da Organização Pan-Americana da Saúde sobre a Promoção e a Publicidade de Alimentos e Bebidas Não Alcoólicas para Crianças nas Américas. Washington: OPAS; 2012.

26. Gelbort J, Henriques I, Miguel JJ. Publicidade de Alimentos e crianças: Regulação no Brasil e no Mundo. São Paulo: Saraiva; 2013.
27. Meller FO, Araújo CLPavin, Madruga SW. Fatores associados ao excesso de peso em crianças brasileiras menores de cinco anos. Cien Saude Colet 2014; 19(3):943955.

28. de Ruyter JC, Olthof MR, Kuijper LD, Katan MB. Effect of sugar-sweetened beverages on body weight in children: design and baseline characteristics of the Double-blind, Randomized INtervention study in Kids. Contemp Clin Trials 2012; 33(1):247-257.

29. Kosova EC, Auinger P, Bremer AA. The relationships between sugar-sweetened beverage intake and cardiometabolic markers in young children. $J$ Acad Nutr Diet 2013; 113(2):219-227.

30. Silva AAM. A pesquisa na área da saúde da criança e as coortes brasileiras. Cien Saude Colet 2010; 15(2):328329.

31. Bray GA, Popkin BM. Calorie-sweetened beverages and fructose: what have we learned 10 years later. Pediatr Obes 2013; 8(4):242-248.

32. Morgan RE. Does consumption of high-fructose corn syrup beverages cause obesity in children? Pediatr Obes 2013; 8(4):249-254.

33. Toloni MHdA, Longo-Silva G, Goulart RMM, Taddei JAAC. Introdução de alimentos industrializados e de alimentos de uso tradicional na dieta de crianças de creches públicas no município de São Paulo. Revista de Nutrição 2011; 24(1):61-70.

Artigo apresentado em 15/05/2014

Aprovado em 02/02/2015

Versão final apresentada em 04/02/2015 